\title{
An adult case of lymphangiomatosis of the mediastinum, pulmonary interstitium and retroperitoneum complicated by chronic disseminated intravascular coagulation
}

\author{
K. Takahashi*, H. Takahashi*, K. Maeda*, S. Homma*, \\ T. Uekusa**, T. Dambara*, S. Kira*
}

\begin{abstract}
An adult case of lymphangiomatosis of the mediastinum, pulmonary interstitium and retroperitoneum complicated by chronic disseminated intravascular coagulation. $K$. Takahashi, H. Takahashi, K. Maeda, S. Homma, T. Uekusa, T. Dambara, S. Kira. ( Journals Ltd 1995.

ABSTRACT: Pulmonary lymphangiomatosis is a rare clinical and pathological entity which is distinct from lymphangiomyomatosis and from pulmonary lymphangiectasis. We report a case of a 20 year old man with diffuse lymphangiomatosis involving the mediastinum, lungs and retroperitoneum.

The patient's intrathoracic lymphangiomatosis produced restrictive and obstructive impairment of his pulmonary function, but did not result in chylothorax or chylopericardium, which are two common manifestations of the disease. The patient's clinical course was remarkable for the concomitant development of chronic disseminated intravascular coagulation (DIC). Lymphangiomatosis involving both the mediastinum and pulmonary parenchyma is rare, and DIC is a very unusual complication of lymphangiomatosis.
\end{abstract}

Eur Respir J., 1995, 8, 1799-1802.
*Dept of Respiratory Medicine, Juntendo University School of Medicine, Tokyo, Japan. **Dept of Pathology, St. Luke's International Hospital, Tokyo, Japan.

Correspondence: K. Takahashi

Dept of Respiratory Medicine, Juntendo University School of Medicine, 2-1-1 Hongo, Bunkyo-ku, Tokyo 113, Japan.

Keywords: Disseminated intravascular coagulation, lymphangiomatosis, mediastinum, pulmonary interstitium, retroperitoneum

Received: January 211995

Accepted after revision May 81995
Lymphangiomas are benign, slow growing lesions characterized by proliferating lymphatic vessels. It is controversial whether they are neoplastic or hamartomatous in origin. They usually occur in the neck, skin or other soft tissues of infants and children. The clinical course of patients with lymphangiomas is relatively benign [1]. Lymphangiomatosis is a rare disorder characterized by multiple lymphangiomas in the liver, soft tissues, bone, spleen, mediastinum, or lungs [2]. This disease is usually fatal in infancy or early childhood, especially when it is associated with other complex pulmonary and cardiac anomalies [3]. The onset of clinical manifestations may be delayed into adolescence or early adulthood in patients with a mild form of the disease [3]. We present the case of a patient with anterior mediastinal lymphangiomaosis involving the great vessels, large airways, heart, and pulmonary parenchyma. This patient also developed disseminated intravascular coagulation (DIC) in association with his lymphangiomatosis.

\section{Case report}

A 20 year old man was admitted to the hospital for further evaluation of mediastinal widening and diffuse interstitial markings on his chest roentgenogram. These abnormalities had first been noticed 5 yrs previously, and had insidiously progressed without any symptoms.

The patient was the product of an uncomplicated pregnancy and delivery, and had experienced no developmental or growth problems. His past medical history was remarkable only for bronchial asthma at 5 yrs of age and rheumatic pericarditis at $10 \mathrm{yrs}$ of age. He had smoked 10 cigarettes $\cdot$ day $^{-1}$ for 4 yrs. No family history of lymphoedema, bleeding tendencies, or vascular malformations was present. The patient's physical examination was completely normal. His respiratory rate was 18 breaths $\cdot \mathrm{min}^{-1}$, blood pressure $125 / 80 \mathrm{mmHg}$; and pulse was 86 beats $\cdot \mathrm{min}^{-1}$. No cutaneous nevi or lymphangiomas were evident. Auscultation of the chest revealed clear breath sounds throughout all lung fields. His cardiac sounds were normal and no murmurs were present. He had no abdominal masses, hepatomegaly, or splenomegaly. No peripheral oedema was present, and his neurological examination was normal.

A chest roentgenogram taken at the time of admission demonstrated cardiomegaly (cardiothoracic ratio $=57 \%$ ), widening of the upper mediastinum, and diffuse interstitial markings in both lung fields (fig. 1). Computed tomography (CT) scan of the chest revealed a large anterior mediastinal mass with fatty attenuation. The mass involved surrounding structures, including the great vessels, large airways, and heart (fig. 2a). No enhancement of the lesion was noted after administration of intravenous contrast. The chest CT scan also demonstrated pulmonary parenchymal perivascular and peribronchial infiltrations, which were most prominent in the proximal region (fig. 2b). An abdominal CT scan revealed extension of 


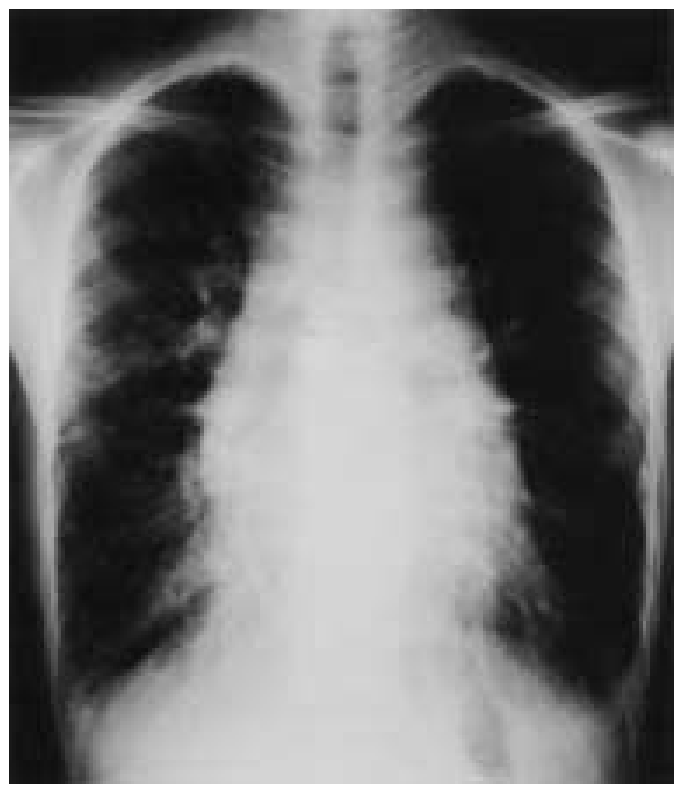

Fig. 1. - Posteroanterior chest roentgenogram on admission showing widening of the mediastinum, enlargement of the cardiac shadow, and increased interstitial markings in both lung fields.

the mediastinal mass into the retroperitoneal space without involvement of the liver or spleen. Echocardiographic examination demonstrated encasement of the heart and great vessels by the lesion. Pulmonary function tests showed combined restrictive and obstructive changes. Vital capacity (VC) was $2.19 \mathrm{~L}$ ( $52 \%$ of predicted value); forced vital capacity (FVC) was $2.15 \mathrm{~L}$; forced expiratory volume in one second $(\mathrm{FEV} 1)$ was $1.45 \mathrm{~L}$; and FEV1/FVC was $67 \%$. These values did not improve after bronchodilator inhalation. With the exception of platelet count and coagulation studies, routine blood tests were all within normal limits: platelet counts, $46 \times 10^{9} \cdot \mathrm{L}^{-1}$; erythrocyte sedimentation rate $2 \mathrm{~mm} \cdot \mathrm{h}^{-1}$; fibrinogen, $1.26 \mathrm{~g} \cdot \mathrm{L}^{-1}$ (normal: $1.5-4.0 \mathrm{~g} \cdot \mathrm{L}^{-1}$ ); fibrin degradation products 21.4 $\mathrm{g} \cdot \mathrm{L}^{-1}$ (normal $<10 \mathrm{~g} \cdot \mathrm{L}^{-1}$ ); soluble fibrin monomer complex, positive; thrombin anti-thrombin complex, 0.0147 $\mathrm{g} \cdot \mathrm{L}^{-1}$ (normal $<0.003 \mathrm{~g} \cdot \mathrm{L}^{-1}$ ). These findings fulfilled the criteria for disseminated intravascular coagulation (DIC) [4]. A bone marrow aspirate demonstrated a slight increase in the number of megakaryocytes.

Ultrasonography-guided tumour biopsy of the anterior mediastinal mass was attempted to obtain a histological diagnosis; however, the specimens were insufficient for analysis. Anterior mediastinotomy under platelet replacement was then performed to obtain material for pathological diagnosis. Minimal chylous fluid was exuded from the mass at the time of this biopsy. Since the anterior mediastinal mass was firmly adherent to adjacent mediastinal structures, it was not possible to dissect the mass from the lung to obtain a specimen of pulmonary parenchyma. Therefore, lung tissue specimens were obtained by transbronchial lung biopsy (TBLB). Histologically, the mediastinal mass was predominantly composed of a proliferation of ectatic lymphatic vessels lined by thin endothelial cells. Adipose tissue and fibrous tissue components a)

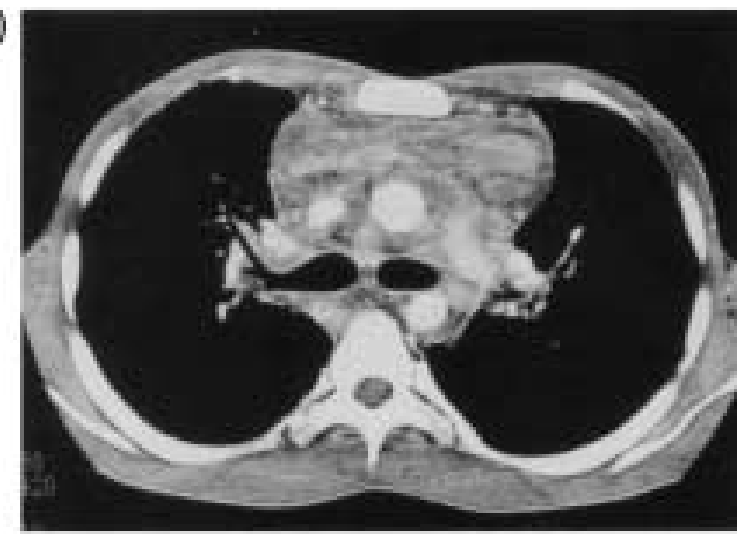

b)

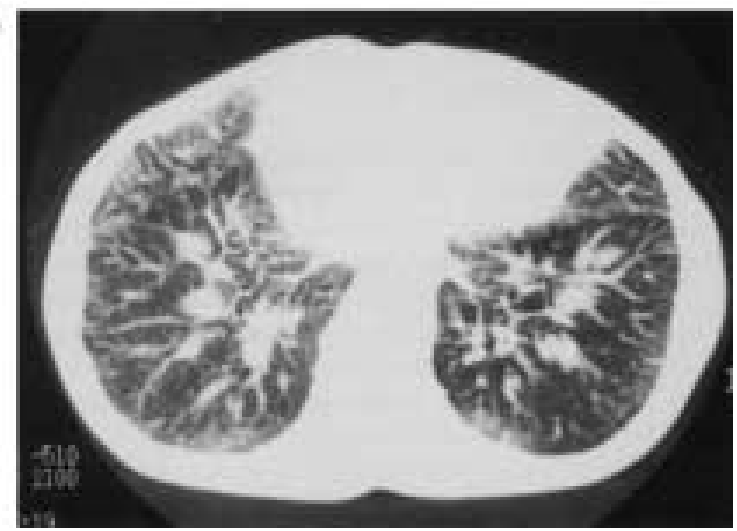

Fig. 2. - a) Computed tomography (CT) scan of the chest with intravenous contrast, demonstrating a large anterior mediastinal mass with fatty attenuation. The mass engulfs the great vessels and large airways. (Window level $80 \mathrm{HU}$; window width $420 \mathrm{HU}$ ). b) CT scan demonstrating diffuse perivascular and peribronchial infiltrations, which are most prominent in the proximal regions. (Window level $-510 \mathrm{HU}$; window width $2,100 \mathrm{HU}$ )

were also observed in the lesion (fig. 3). The specimens of lung parenchyma revealed similar findings to those of the mediastinal mass, in which variably sized endothelial-lined spaces were observed in the peribronchial and interlobular interstitium. No proliferation of smooth muscle cells

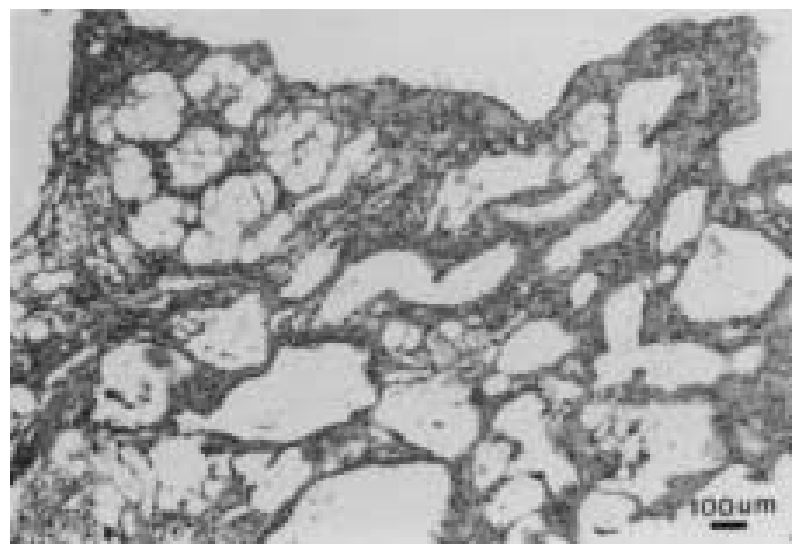

Fig. 3. - The microscopic appearance of the anterior mediastinal mass, showing proliferation of ectatic lymphatic vessels. Components of adipose and fibrous tissue are also seen (Elastica van-Gieson stain: magnification $\times 40$; internal scale bar $=100 \mu \mathrm{m}$ ). 
was noted. None of the cells lining the spaces stained with an antibody to factor VIII on immunohistochemical analysis, suggesting that the lesion was not composed principally of blood vessels. A diagnosis of lymphangiomatosis was made based on these histological findings and the extent of the lesions as assessed by roentgenographic studies and mediastinotomy.

Because the patient was asymptomatic and his DIC had not worsened as assessed by blood tests, we elected to observe him without any specific treatment. He has been followed for more than $1.5 \mathrm{yrs}$ and remains free from any symptoms. He has not developed dyspnoea on exertion, lymphoedema, or bleeding. Furthermore, his chest roentgenographic findings have remained stable.

\section{Discussion}

Lymphangiomas are benign lesions characterized by proliferating lymphatic vessels, and usually occur in the neck, axillae, or other soft tissues in newborns and infants [1]. Lymphangiomatosis is defined as a unique pathological condition in which multiple lymphangiomas are present, most characteristically in the liver, spleen, mediastinum, lungs, or soft tissues. It is a rare disorder, occurring mainly in infants and children; it seldom affects patients older than 20 yrs of age [1]. The aetiology of lymphangiomatosis remains controversial. The generalized distribution of the disease is more suggestive of lymphatic malformation or maldevelopment than neoplasia. A hamartomatous origin of the disease cannot be excluded because disorganized lymphatic structure is occasionally observed in the lymphangiomas [5, 6]. The histological findings of the present case may also support the concept of a hamartomatous process, since several components, such as adipose and fibrous tissue, were mixed in the proliferation of lymphatic vessels.

We present this case as an example of primary intrathoracic lymphangiomatosis involving the mediastinum and lung parenchyma. Although the predominant site of the mediastinal lesion was the anterior mediastinum, the lesion involved surrounding structures including the heart, great vessels, and large airways. The lesion also extended below the diaphragm into the retroperitoneum. The roentgenographic finding, in conjunction with the characteristic histological picture, confirmed the diagnosis of mediastinal lymphangiomatosis.

The differential diagnosis of bilateral diffuse lung infiltrates in this case includes lymphangioleiomyomatosis (also known as lymphangiomyomatosis or LAM [7]), primary lymphangiectasis, and diffuse pulmonary lymphangiomatosis. Lymphangioleiomyomatosis is a distinct entity, in which the predominant feature is interstitial smooth muscle cell proliferation in the pulmonary parenchyma [8]. These histological features are absent in the present case. Furthermore, the diagnosis of lymphangioleiomyomatosis could easily be excluded in this patient because it occurs only in females in their reproductive years. The distinction between primary lymphangiectasis and diffuse pulmonary lymphangiomatosis is usually made on the basis of the size and number of participating lymphatic spaces [7, 9]. In lymphangiectasis, the spaces tend to be larger and the mesenchymal tissue component is less pronounced $[7,9]$. In the present case, differentiation between these two disorders based solely on histology was difficult, mainly because the pulmonary parenchymal specimens obtained by TBLB were fairly small. Nevertheless, primary pulmonary lymphangiectasis was an unlikely diagnosis in this case, since it occurs almost exclusively in infants and young children, and it is uniformly fatal [3,9]. Consequently, we believe that the pulmonary lesions of the present case resulted from primary lymphangiomatosis with secondary lymphangiectasis of intrapulmonary lymphatic structures.

It is unclear whether the mediastinal and lung lesions represent spread of the disease from one structure into an adjacent one, or if they represent multicentric origins of disease. Both lesions were evident on the patient's initial chest roentgenogram 5 yrs previously. This patient's presentation is unusual and unique compared to that of other patients with lymphangiomatosis because of the simultaneous involvement of the lungs and mediastinum, the absence of chylous pleural or pericardial effusions, and the presence of haematological evidence of DIC. Despite the anatomical proximity of the lungs and mediastinum, simultaneous occurrence of mediastinal and pulmonary lymphangiomatosis is rare. Of 14 patients with mediastinal lymphangiomas (including lymphangiomatosis) reported by BRown et al. [10], one patient had disease involving the lung in which the right paratracheal mass directly extended to right lung apex. TAZELAAR et al. [7] reported nine patients with diffuse pulmonary lymphangiomatosis, and none had mediastinal involvement. To our knowledge, only three patients with diffuse pulmonary lymphangiomatosis have been reported to have mediastinal involvement $[3,11]$. In these patients, the mediastinum appeared normal radiographically, but the mediastinal lymphangiomatosis was diagnosed either by open biopsy or autopsy. It is possible that subclinical mediastinal involvement is relatively common in diffuse pulmonary lymphangiomatosis, despite normal roentgenographic analysis of the mediastinum.

Lymphangiomas are localized lesions that are thought to arise from sequestered lymphatics that fail to communicate normally with the lymphatic system [7]. They are usually cured by surgical resection, and the prognosis seems to be excellent. In contrast, the most significant adverse prognostic factor in lymphangiomatosis is the involvement of serosal surfaces and viscera [11]. Most patients with mediastinal and pulmonary lymphangiomatosis develop chylothorax or chylopericardium $[3,7$, 11 ], suggesting that these lesions communicate with major lymphatic trunks. Interestingly, the patient presented in this report did not develop chylothorax, chylopericardium, or lymphoedema, despite extensive involvement of the mediastinum and lungs. Furthermore, minimal chylous fluid exuded during the mediastinotomy. These observations suggest that this patient's lesions are discrete and isolated from the major lymphatic system. The patient has not developed progressive pulmonary or pericardial restriction. 
Coagulopathy has previously been reported in association with lymphangiomatosis, and several potential mechanisms for this association have been advanced [12, 13]. Local production of fibrinolysin by lymphangiomas may be responsible for the coagulopathy [12]. Focal consumption of fibrinogen and platelets in a diseased spleen and liver triggered by the infusion of platelets for an external injury has also been reported as a possible mechanism [13]. In the present case, we have excluded other possible causes of DIC, such as thrombotic thrombocytopenic purpura (TTP), primary haematopoietic disorders, and Kasabach-Merritt syndrome. Therefore, the DIC in this patient may be a complication of generalized lymphangiomatosis. The persistent thrombocytopenia in this patient will necessitate careful clinical follow-up.

Acknowledgement: The authors thank K. Tanabe, Department of Surgery, Massachusetts General Hospital, for critical review of the manuscript.

\section{References}

1. Marchevsky AM, Kaneko M. Mesenchymal tumor of the mediastinum. In: Surgical pathology of the Mediastimum. New York, Raven Press, 1984; pp. 235-238.

2. Gilsanz V, Yer HC, Baron MG. Multiple lymphangiomas of the neck, axillae, mediastinum and bones in an adult. Radiology 1976; 120: 161-162.

3. Swank DW, Hepper NGG, Folkert KE, Colby TV.
Intrathoracic lymphangiomatosis mimicking lymphangioleiomatosis in a young woman. Mayo Clin Proc 1989; 64: 1264-1268.

4. White GC II. Approach to the bleeding patient. In: Colman RW, et al., eds. Hemostasis and Thrombosis: Basic Principles and Clinical Practice. 2nd Edn. Philadelphia, JB Lippincott 1987; 1048-1060.

5. Muhammad AKB, John WF, Isac G, John CN. Pleuropulmonary and skeletal lymphangiomatosis with chylothorax and chylopericardium. Ann Thorac Surg 1985; 40: 398-401.

6. Godart S. Embryological significance of lymphangioma. Arch Dis Child 1966; 41: 204-206.

7. Tazelaar HD, Kerr D, Yousem SA, Saldana MJ, Langston C, Colby TV. Diffuse pulmonary lymphangiomatosis. Hum Pathol 1993; 24: 1313-1322.

8. Taylor JR, Ryu J, Colby TV, Raffin TA. Lymphangioleiomyomatosis. Clinical course in 32 patients. $N$ Engl $J$ Med 1990; 323: 1254-1260.

9. Wagenaar SJ SC, Swierenga J, Wagenvoort CA. Late presentation of primary pulmonary lymphangiectasis. Thorax 1978; 33, 791-795.

10. Brown LR, Reiman HM, Rosenow III RC, Gloviczki PM, Divertie MB. Intrathoracic lymphangioma. Mayo Clin Proc 1985; 61: 882-891.

11. Ramani P, Shah A. Lymphangiomatosis: histologic and immunohistochemical analysis of four cases. Am J Surg Pathol 1993; 17: 329-335.

12. Diez WH, Stuart MJ. Splenic consumptive coagulopathy in a patient with disseminated lymphangiomatosis. J Pediatr 1977; 421-423.

13. Thomas K, Leung A, Lynn J. Abdominal cystic lymphangiomatosis: report of a case and review of the literature. Br J Radiol 1985; 58: 467-469. 